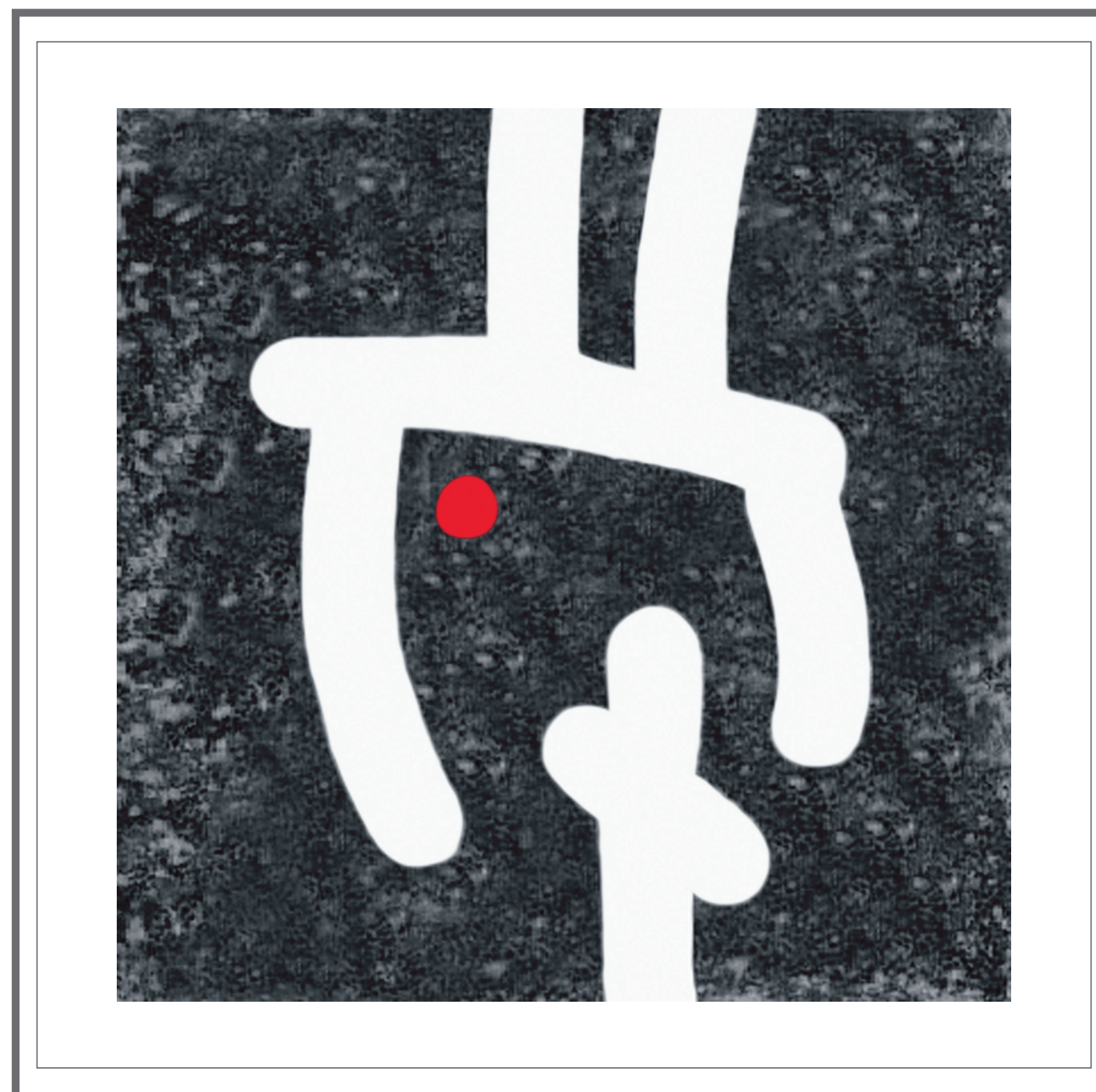

(Arte)

Estampa Digital

Título:

Colgante I 


\section{Autor:}

Luis Fernando Luna

Técnica:

Impresión Digital Giclée sobre papel de Algodón 255 gr.

Medida: 40 X $40 \mathrm{~cm}$ (Estampa 30 X $30 \mathrm{~cm}$ )

Edición de 25 ejemplares 


\title{
LA ESTABILIZACIÓN SOCIOECONÓMICA Y EL DERECHO A LA EDUCACIÓN DE LA POBLACIÓN EN SITUACIÓN DE DESPLAZAMIENTO FORZADO EN EL ÁREA METROPOITANA DE BUCARAMANGA (AMB) ${ }^{1}$
}

\section{SOCIO-ECONOMIC STABILIZATION AND THE RIGHT TO EDUCATION OF DISPLACED POPULATION IN THE METROPOLITAN AREA OF BUCARAMANA (AMB)}

\section{Gonzalo Alberto Patiño Benavides ${ }^{2}$} Óscar Herrán Falla

\begin{abstract}
Resumen
En este trabajo se aborda el componente del derecho a la educación en el contexto de los cambios y transformaciones del ente territorial en Santander. La Recuperación Temprana (RT), entendida como la capacidad de restaurar, tanto las instituciones nacionales como las de sus comunidades frente a conflictos o desastres naturales, abarca un proceso multidimensional guiado básicamente por los principios humanitarios. Se trata, por lo tanto, de generar y reforzar procesos de desarrollo social y económicamente sostenibles en fases de pos-crisis, que refuercen capacidades propias en una amplia gama de servicios y aspectos, que normalmente contemplan, entre otros, la reintegración de las personas en situación de desplazamiento.
\end{abstract}

Palabras clave: Estudios Humanitarios, Capacidades, Desarrollo, Estabilización Socioeconómica, Recuperación Temprana

\begin{abstract}
This paper discusses briefly the component of the right to education in the context of changes and transformations of the local authority in Santander. Early Recovery (RT), defined as the ability to restore both national institutions and those of their communities from conflict or natural disasters, covering a multidimensional process guided primarily by humanitarian principles. It is, therefore, to generate and reinforce processes of social and economically sustainable postcrisis phases, to strengthen their own capacities in a wide range of services and aspects, which usually include among others the reintegration of people displacement
\end{abstract}

Key Word: Humanitarian Studies, capabilities, development, economic stabilization, early recovery

1 Artículo resultado del proyecto "Evaluación de impacto de estabilización socioeconómica de la población en situación de desplazamiento forzado en el Área Metropolitana de Bucaramanga", que se ejecutó entre los años 2010 y 2011 con recursos de la UIS, con el aval y participación de los miembros del Nodo del Instituto de Estudios Humanitarios (IEH) en Bucaramanga

2 Economista, Ph. D. Profesor Asociado Tiempo Completo, Escuela de Economía y Administración Universidad Industrial de Santander. Coordinador Punto Focal de Información, Nodo IEH- Bucaramanga. Centro de Investigaciones Epidemiológicas, Observatorio Epidemiológico de Enfermedades Cardiovasculares, Bucaramanga

3 Nutricionista Dietista, Magíster en Epidemiología. Profesor Titular Laureado, Escuela de Nutrición y Dietética, Universidad Industrial de Santander, Centro de Investigaciones Epidemiológicas, Observatorio Epidemiológico de Enfermedades Cardiovasculares, Bucaramanga. 


\section{La educación, las capacidades humanas y el desarrollo}

Entender el proceso educativo significa siempre tender un puente con el pasado e intentar comprender el legado cultural que otras sociedades nos han dejado: un acervo de conocimientos exquisito que ha permitido ser lo que realmente somos. A la conservación y renovación de esta experiencia se han dedicado las mentes más ilustres desde tiempos remotos que recrean y promueven hábitos que han dejado una huella profunda en la humanidad. Pero, es precisamente la actividad humana la que ha permitido el desarrollo de una función creadora, que prácticamente no conoce hoy límites, ante los avances inobjetables de la ciencia, la técnica y la tecnología. Para Vigotsky, la actividad creadora de la imaginación cumple un papel esencial, pues:

“(..) se encuentra en relación directa con la riqueza y diversidad de la experiencia
acumulada por el hombre, porque esta experiencia ofrece el material con el que
erige sus edificios de fantasía. Cuanto más rica sea la experiencia humana, tanto
mayor será el material del que dispone esa imaginación. Por eso, la imaginación
del niño es más pobre que la del adulto, por ser menor su experiencia”(Vigotsky,
2005 p 19).

En la perspectiva de Castoriadis, esta actividad creadora del ser humano termina proyectándose a través de un proceso de discontinuidad y ruptura en el que se da el paso fundamental de sociedades heterónomas -aquellas que imputan a otro la creación de sus instituciones, de su nomos- a sociedades donde las significaciones sociales imaginarias correspondientes dan paso al reconocimiento de una dimensión subjetiva o del para sí. Se trata, no sólo de la necesidad del ser humano viviente de crear instituciones imaginarias, sino del hecho esencial que el individuo elabore su propio proyecto de autonomía. Estamos ante tres ideas principales: primera, lo viviente es para sí en tanto que es finalidad; segunda, crea cada vez su mundo propio; tercera, este mundo propio es un mundo de representaciones, de afectos y de intenciones (Castoriadis,2005).

No estamos muy lejos, por cierto, del tradicional concepto de libertad de Mill, cuando expresaba que la libertad humana exige libertad en nuestros gustos y en la determinación de nuestros propios fines; libertad para trazar el plan de nuestra vida según nuestro propio carácter para obrar como queramos, sujetos a las consecuencias de nuestros actos (Mill, 2001 p 32).

La filosofía política contemporánea ha puesto en un punto más alto esta discusión, al centrarse en un conjunto de libertades humanas en una sociedad bien ordenada, cuya estructura básica reposa en la idea de un sistema equitativo de cooperación social en el que se asignan derechos y deberes y al cual se llega mediante un consenso entrecruzado. Esta es una facultad también creadora del hombre, que se concibe a sí mismo con ciertas facultades morales. Se trata de la justicia como equidad pensada, como es apenas lógico suponer, para una sociedad democrática. Se reconoce 
enseguida la existencia de desigualdades sociales y económicas aceptables dentro de un cierto rango de diferencias. Sin embargo, lo que hace ralamente atractiva esta distinción es la posibilidad de acceso que tienen los seres humanos a un paquete básico de libertades iguales para todos o "igualdad equitativa de oportunidades". Las desigualdades sociales y económicas -señala Rawls- tienen que satisfacer dos condiciones: en primer lugar, tienen que estar vinculadas a cargos y posiciones abiertos a todos en condiciones de igualdad equitativa de oportunidades y, en segundo lugar, las desigualdades deben redundar en un mayor beneficio de los miembros menos avanzados de la sociedad (principio de diferencia) (Rawls, 2005 :17).

A partir de allí el filósofo norteamericano precitado, se introduce al concepto de bienes primarios - criticados por Sen, distinguiéndose los siguientes: los derechos y libertades básicos (libertad de pensamiento y libertad de conciencia), la libertad de movimiento y libre elección del empleo, los poderes y las prerrogativas que acompañan a cargos y posiciones de autoridad y responsabilidad, ingresos y riqueza, como medios de uso universal. Nuestro tercer personaje Sen - conocido también como el Nobel de los pobres, centra su atención en el concepto de libertad como desarrollo, que abarca

"Tanto los procesos que hacen posible la libertad de acción y decisión como las oportunidades reales que tienen los individuos, dadas sus circunstancias personales y sociales". (Sen, $2000 \mathrm{p} 33)$

Tradicionalmente, la economía ha centrado su atención en las relaciones existentes entre educación y crecimiento económico, como senda segura que permite el bienestar y el desarrollo de una nación; en particular a través de una senda de cambio técnico y tecnológico. (Sala i Martin, 1999 :13). Estas observaciones, es preciso señalar, se derivan de los trabajos seminales de Solow en términos del papel de la inversión y el crecimiento endógeno (Solow, 1992). Las críticas, en nuestro país, a la propuesta neoclásica y su perspectiva de crecimiento económico basada en mayores niveles de apertura económica, han apuntado a señalar, que:

"El aumento de la tasa de crecimiento está asociado con un mayor crecimiento del capital y del trabajo, dejando en claro que el cambio tecnológico es pequeño $y$ de naturaleza exógena. Por el contrario, en los países desarrollados el capital físico es más complementario del capital humano y, en general, de la tecnología" (Sarmiento, 2000 p 17).

En este sentido, es válida la sentencia de Naredo cuando afirma:

"Hacemos caso omiso de la distinción entre crecimiento y desarrollo en una disquisición verbal vacía de contenido, aireada a veces por una izquierda deseosa de marcar diferencias respecto a la derecha, cuando ambas aparecen hermanadas en los objetivos últimos desarrollistas" (Naredo, 1996 p 36) 
Para Sen, sin embargo, es necesario introducir otros referentes de desarrollo y bienestar. Señala, el "papel constitutivo" de la libertad, que se asocia necesariamente con un conjunto de libertades fundamentales para la calidad de vida humana (evitar privaciones como la inanición, la desnutrición, morbilidad evitable, mortalidad prematura, o libertad relacionada con la capacidad para leer, escribir, participar o capacidad de disentir políticamente, entre otras). El "papel instrumental", se relaciona con los distintos tipos de derechos y oportunidades que permiten expandir la libertad del hombre en general (desarrollo): libertades políticas, servicios económicos, garantías de transparencia, seguridad protectora, oportunidades sociales. La expansión de las oportunidades sociales a través del gasto público social se constituye en un referente importante para medir el alcance de las políticas públicas, traducidas en una mayor esperanza de vida y reducción de tasas de mortalidad, entre otros objetivos. En este sentido, la educación se constituye en un elemento indispensable a la hora de evaluar las posibilidades que nos brinda una política pública acertada en relación con la población más vulnerable, en particular en momentos en que se afianzan los procesos de globalización económica, pues como afirma Bauman:

"la globalización divide en la misma medida que une (...) justamente con las dimensiones emergentes, y planetarias de los negocios, las finanzas, el comercio y el flujo de información, se pone en marcha un proceso "localizador" de fijación del espacio (Bauman, 1999 p 8)

Por último, conviene señalar los aportes de Martha Nussbaum. En esencia, esta autora parte de una premisa sencilla, al reconocer la dificultad de pensar los problemas de la justicia social en Occidente, pues parten de una unidad básica como es el Estado-nación. De allí su consideración de abordar los tres problemas no resueltos por la teoría contractualista, reconocidos incluso por Rawls. Con ello pretende defender que la versión del "enfoque de las capacidades", aporta mejores ideas a la tradición del contrato social. Esto es especialmente importante en relación con las personas que se encuentran en una posición asimétrica en la sociedad (niños, mujeres y adultos mayores) pues, en sus palabras, “... se hallan ipso facto ausentes del grupo de ciudadanos para quienes se diseñan los principios” (Nussbaum, 2007 $: 51)$.

Es decir, el punto de partida de Nussbaum es la valoración de lo que son realmente capaces de ser y hacer las personas con un conjunto determinado de bienes y no solamente como se sienten en relación con ellos. Se trata -como ella lo señala-, de una descripción de los derechos mínimos - capacidades humanas básicas- compatibles con diferentes respuestas sobre cuestiones de justicia y distribución. La mayoría de estas capacidades se conecta de alguna manera con la educación, pero quisiéramos resaltar aquí la cuarta: sentidos, imaginación y pensamiento, pues se ubica mejor en la perspectiva que este trabajo pretende desarrollar y ubica a la educación como su núcleo central: 
"Poder usar los sentidos, la imaginación el pensamiento y el razonamiento, y hacerlo de un modo "auténticamente humano», un modo que se cultiva y se configura a través de una educación adecuada, lo cual incluye la alfabetización y la formación matemática y científica, aunque en modo alguno se agote en ello" ((Nussbaum, 2007 p 88).

Nos asalta, pues, una inquietud ante estas primeras reflexiones: ¿Qué tipo de sendero o huellas permanentes han dejado en los niños, niñas y adolescentes de Colombia esta guerra perpetua, catalogada de tiempo atrás como uno de los conflictos armados más prolongados de la historia? ¿Qué significa para ellos una vida desprovista - para retomar la idea original de Nussbaum- de educación? ¿Qué significa en este contexto la Recuperación Temprana? No pretendemos aquí dar una respuesta a estos interrogantes, más bien quisiéramos pensar que es en la educación como derecho esencial, en la que se abre un conjunto de posibles respuestas. Un medio transformador de experiencias que debe permitir -en principio- un conjunto de oportunidades para una mejor calidad de vida o, si se quiere, capaz de formar una imagen del mundo distinta a la que ha otorgado al país el conflicto armado. La educación se constituye en el medio ideal en el que se hace posible una simbiosis particular entre experiencia, método y ensayo (Morin, 2008)

Más allá de su pasado -o de su precario presente-, los niños, niñas y adolescentes que han tenido que soportar las ataduras de la guerra, exigen de nuestra sociedad y sus instituciones bases suficientemente sólidas para su actividad creadora en el futuro, como elemento vital, que les permita restituir su confianza en su propia autenticidad. Se debe adelantar, sin embargo, una consideración preliminar antes de aventurarnos a interrogar en qué medida nuestra sociedad ha logrado este objetivo. La investigación apunta, inequívocamente a una dirección: persiste un conjunto de brechas a nivel territorial entre el derecho formalmente reconocido y los hechos sociales, que constriñe la posibilidad de un desarrollo humano digno para un amplio conjunto de la población en situación de desplazamiento forzado. Los deberes mínimos del Estado frente a los niños, niñas y adolescentes de estos hogares comprometen seriamente la estabilidad futura de sistemas sociales anclados, de tiempo atrás, en una subordinación inexplicable a las fuerzas del mercado. Se devela aún, incluso en el plano territorial, la persistencia de un orden económico y social injusto y devastador que compromete buena parte de los exiguos logros que para el resto de la población se han alcanzado en materia educativa.

Y esta es una de las manifestaciones más palmarias de los efectos perversos del conflicto armado en Colombia, pues radica en la ruptura del proceso educativo al que se ven sometidos niñas, niños y adolescentes en sus distintas etapas de desarrollo, sometidos a una doble condición de desigualdad y ampliada hasta el límite, por lo tanto, la cadena de injusticias que arrastran sus núcleos familiares, una vez han se han visto obligados a abandonar las zonas rurales de las que regularmente provienen. Situados de manera precaria en los centros urbanos y sus áreas metropolitanas, 
enfrentan el reto de asumir con dignidad el proceso de adaptación a las exigencias de un entorno que es regularmente hostil. La respuesta de los entes gubernamentales e institucionales se ha centrado de tiempo atrás en proveer una política pública, que permita una adecuada inserción al sistema escolar tradicional, al ofrecer facilidades de acceso y generar los dispositivos necesarios para que los entes territoriales asuman a nivel local los mandatos legales de manera eficaz, así como las exigencias de la Corte Constitucional, en el sentido de superar el estado de cosas inconstitucional (ECI), que prevalece en relación con la población en situación de desplazamiento forzado. Un asunto central de esta política es la estabilización socioeconómica.

\section{La educación en la estabilización socioeconómica de la pobla- ción en situación de desplazamiento forzado, según las $\mathbf{E N V}^{4}$}

La estabilización socioeconómica de las personas en situación de desplazamiento forzado suele comprenderse en un marco amplio de acciones que, en el tiempo, se desarrollan bajo el amparo del Estado, y que propenden por una real protección de quienes se han visto obligados, por diversas razones, a abandonar sus lugares de residencia habitual. Sin embargo, las razones de Estado -en términos de Foucaultnos obligan a pensar todavía la perpetuidad del conflicto armado interno en distintos ámbitos: primero, como producto de un conjunto de violaciones sistemáticas a las libertades fundamentales, que perpetúan un Estado de Cosas Inconstitucional (ECI) como lo definió la Sentencia T-025 del 2004; segundo, como una estrategia de dominio territorial por parte de los actores armados. En ambos casos se evidencia una sistemática profundización de las desigualdades existentes y un desconocimiento de las diferencias culturales que subyacen en nuestro territorio a la hora de abordar posibles salidas al conflicto con graves repercusiones en el campo humanitario.

En este sentido, la Corte Constitucional frente a los altos índices de informalidad y bajos ingresos que presentan las personas desplazadas, adoptó un conjunto de indicadores de goce efectivo que permitiese en el corto y mediano plazo consolidar la estabilización socioeconómica. En particular, en lo que atañe a la generación de ingresos se adoptaron los siguientes indicadores: primero, el hogar tiene al menos una fuente de ingreso autónoma y su ingreso supera como mínimo la línea de indigencia; segundo, el hogar tiene como mínimo una fuente de ingreso autónoma y su ingreso se ubica por encima de la línea de pobreza. Resulta evidente, así las cosas, que los resultados expuestos tanto en la Primera como en la Tercera Encuesta Nacional de Verificación (I y III ENV) apuntan a un alto índice de pobreza e indigencia por parte

4 Durante la investigación, se aplicó una encuesta a 272 familias que viven en situación de desplazamiento forzado en los municipios de Bucaramanga, Floridablanca y Piedecuesta (Santander, Colombia). La encuesta se realizó durante el segundo semestre del 2010. El responsable del hogar respondió una serie de preguntas, según los parámetros de las encuestas nacionales de verificación en relación con el goce efectivo de derechos. 
de las personas en situación de desplazamiento. En otras palabras, la gran mayoría de los hogares no cumple con los indicadores, pues, según la primera encuesta, sólo el $2 \%$ supera la línea de pobreza y un $26 \%$ está bajo la línea de indigencia o sea de pobreza extrema. Los resultados no cambian sustancialmente en la tercera encuesta: sólo el 2,6\% de los desplazados inscritos en el Registro Urbano de Población Desplazada (RUPD), supera la línea de pobreza, mientras que entre los no inscritos, la cifra es del $4,3 \%$.

Esta situación de precariedad extrema, refleja la carencia de una política pública que permita una acción colectiva más coordinada en materia de generación de ingresos. De otra parte, la gravedad de esta situación radica, precisamente, en que se limitan sustancialmente otros derechos como la educación, muy a pesar de los esfuerzos del Estado en materia de gratuidad para esta población. Igualmente, cabe resaltar -como se desprende de la I ENV- que aproximadamente el 25,4\% de la población desplazada en edad de trabajar y que están incluidos en el RUPD, labora en jornadas inferiores a 40 y 48 horas semanales, un $23,9 \%$ trabaja las horas semanales legales, mientras que un $50,7 \%$ excede el tiempo en horas laborales legales. Una discontinuidad y ruptura que se mantiene en la III ENV como se observa a continuación.

Cuadro 1: Distribución de la población desplazada incluida en el RUPD que está ocupada según duración de la jornada laboral

\begin{tabular}{cccc}
\hline $\begin{array}{c}\text { DURACIÓN JORNADA LABORAL } \\
\text { (horas semanales) }\end{array}$ & I ENV & \multicolumn{2}{c}{ III ENV } \\
\cline { 3 - 4 } & & RUPD & NO RUPD \\
\hline Menos de 20 & 11,1 & 11,9 & 10,5 \\
Entre 20 y 39 & 14,3 & 21,5 & 17,7 \\
Entre 40 y 48 & 23,9 & 27,8 & 26,4 \\
Entre 49 y 60 & 22,6 & 20,4 & 23,1 \\
Más de 60 & 28,1 & 18,4 & 22,3 \\
\hline
\end{tabular}

Fuente: En base a los resultados obtenidos por la I ENV-2007 y la III ENV-2010 comisión de seguimiento CID-UN

Un aspecto fundamental de la estabilización socioeconómica viene determinado por el tipo de relación laboral, con el cual se pretende medir la proporción de población ocupada asalariada que tiene contrato de trabajo escrito; en otras palabras, busca establecer la legalidad de la relación contractual. Como se observa en el siguiente Cuadro un mínimo porcentaje $(5,2 \%)$ de la población que aquí se analiza cuenta con un contrato escrito a término indefinido, mientras que el $4 \%$ mantiene contrato a término fijo $(5,7 \%$ y $6 \%$ respectivamente en la IIIENV), en tanto que una gran 
mayoría ( $90 \%$ en la I ENV y $86,6 \%$ en la III ENV)) permanece sin contrato. Es preciso señalar que, estar o no escrito en el RUPD, no marca una gran diferencia en este aspecto.

Cuadro 2: Distribución de la población desplazada, según tipo de contratación por condición de inscripción en el RUPD

\begin{tabular}{lccc}
\hline \multirow{2}{*}{ TIPO DE CONTRATACIÓN } & \multirow{2}{*}{ I ENV } & \multicolumn{2}{c}{ III ENV } \\
\cline { 3 - 4 } & & RUPD & NO RUPD \\
\hline Contrato escrito a término indefinido & 5,2 & 5,7 & 5,5 \\
Contrato escrito a término fijo & 4 & 6 & 4,7 \\
Sin contrato escrito & 90 & 86,6 & 86,1 \\
No sabe si tiene contrato escrito & $*$ & 1,7 & 0,6 \\
\hline
\end{tabular}

Fuente: En base a los resultados obtenidos por la I ENV-2007 y la III ENV-2010 Comisión de Seguimiento CID-UN

El tema de la vulnerabilidad social de niños, niñas y adolescentes en situación de desplazamiento y sus posibles alternativas de solución integral, no puede escapar a las distintas lógicas de intervención, en particular de aquellos actores institucionales que con sus esfuerzos pretenden lograr una adecuada estabilización socioeconómica de estos grupos poblacionales. Uno de los aspectos destacados por la Comisión de Seguimiento a la Política Pública en la primera, segunda y tercera Encuesta Nacional de Verificación, es la alta participación (55,1\%) de niños, niñas y adolescentes en el total de la población desplazada inscrita en el RUPD, así como en la no inscrita $(51,6)$ que tiene menos de dieciocho años de edad, frente al $37 \%$, que representa la población total en ese mismo rango de edad, según el censo del año 2005. Esta condición demográfica, afecta sensiblemente la prevalencia de sus derechos, tanto en el corto como en el mediano plazo, generándose una espiral negativa, que se extiende a otros ámbitos en los que su participación se ve seriamente comprometida, en particular en relación a sus posibilidades de acceso al sistema educativo.

Para evaluar el goce efectivo del derecho a la educación entre la población desplazada las ENV toman como referencia el Auto No. 109 de 2007 mediante el cual la Corte Constitucional adoptó el indicador de goce efectivo del derecho: Todos los niños y jóvenes del hogar entre 5 y 17 años de edad asisten regularmente a un nivel de educación formal. Para hacer seguimiento y medir el grado de avance, se adoptó el indicador complementario: porcentaje de niños desplazados incluidos en el RUPD que asisten a un establecimiento educativo y el Indicador asociado: porcentaje de niños incluidos en el RUPD que son beneficiados con medidas de acompañamiento 
a la permanencia escolar. La Corte Constitucional adoptó, igualmente, mediante el Auto 116 de 2008 un conjunto de indicadores complementarios y sectoriales, que se establecen en la II ENV y que se mencionan a continuación:

\section{Indicadores Complementarios}

- Tasa de cobertura neta de educación de población por nivel educativo y desagregado por motivos de discriminación prohibidos.

- Tasa de aprobación de los estudiantes de la población desplazada entre los 5-17 años de edad por nivel educativo.

Indicadores sectoriales asociados

- Proporción de estudiantes provenientes de hogares desplazados que no sufragan ningún costo de la canasta educativa en el nivel básico (derechos académicos, derechos de matrícula, pensiones, uniformes, útiles, transporte escolar).

- Grado de suficiencia de docentes o de su tasa de asistencia a las clases: nivel de instalaciones adecuadas (baños, aulas y demás) en los colegios a los que asisten niños de la población desplazada. (Ver más II encuesta).

Los resultados se materializan en las ENV de la siguiente manera:

Primero, en términos de Acceso, se reconoce el avance en materia de cobertura, pero se observa como insuficiente para lograr el pleno derecho a la educación desde el punto de vista de este criterio. Para el año 2007, la tasa de cobertura bruta en la población desplazada incluida en el RUPD a nivel preescolar es de 56,3\%, lo cual hace suponer que, por distintas barreras, los niños más pequeños se encuentran a cargo de un adulto (padre, madre u otro adulto). En primaria la tasa es superior al $100 \%$ debido a las tasa de extra-edad. Se observan bajas tasas de escolarización en secundaria $(72 \%)$ y en educación media $(31,2 \%)$, panorama lejano a la universalización que se pretende. En la segunda encuesta se señala: la Tasa de Cobertura Bruta (TCB) para los niños entre 5-17 años inscritos en el RUPD es del 90,6\% para el conjunto de los niveles. Para los desplazados no inscritos esta tasa es del $82,6 \%$. Por niveles se observa una tendencia similar a lo encontrado en la primera; es decir, en pre-escolar (más del 50\%, es decir, la mitad de los inscritos y no inscritos en el RUPN no tienen garantizado el acceso en el momento adecuado. Los resultados para la primera y tercera encuesta se muestran a continuación. 
Cuadro 3. Tasa bruta de escolarización de la población desplazada de 5 años o más según nivel educativo, por condición de inscripción en el RUPD

\begin{tabular}{|l|c|c|c|}
\hline \multirow{2}{*}{ NIVEL EDUCATIVO } & \multicolumn{3}{c|}{ TBN } \\
\cline { 2 - 4 } & \multirow{2}{*}{ I ENV } & \multicolumn{2}{c|}{ III ENV } \\
\cline { 3 - 4 } & & RUPD & NO RUPD \\
\hline Preescolar & 56,3 & 52,1 & 53,5 \\
\hline Primaria & 123,4 & 122,7 & 115,9 \\
\hline Secundaria & 72 & 93,1 & 84,3 \\
\hline Media & 31,2 & 43,9 & 43,2 \\
\hline Total Preescolar, Básica y Media & 83,6 & 91,6 & 87,8 \\
\hline
\end{tabular}

Fuente: En base a los resultados obtenidos por la I ENV-2007 y la III ENV-2010 Comisión de Seguimiento CID-UN

Segundo, la Obligatoriedad en el acceso se estima a partir de la tasa neta de escolarización: en preescolar es de 53,5\%; es decir, cerca de la mitad de los niños que deben asistir a este nivel no lo hacen, en otras palabras, gran parte de los niños entre 5-6 años se encuentran por fuera del sistema educativo. En secundaria y media la situación es más compleja; allí se observan tasas netas de escolarización del 49,5\% y del $16,5 \%$ respectivamente o, lo que es lo mismo, uno de cada dos niños entre los 12 y los 15 años de edad no asiste a este nivel

Tercero, la Accesibilidad. La gratuidad se ha convertido en un pilar de la política educativa a nivel nacional. Sin embargo, en el grupo de los niños entre 5-17 años edad, sólo el 66,5\% no debe realizar ningún pago. La matrícula en secundaria y media debe pagarla el 37\% de los niños. Otras fuentes de egresos en el sistema escolar que se constituyen en barreras para el acceso a la educación, son los distintos elementos exigidos en las instituciones como uniformes, cuadernos, elementos de aseo y otros. En el Cuadro 15 se muestra los resultados de los pagos realizados por los hogares de la población desplazada con niños entre 5 y 17 años de edad, según la comparación entre la I y III ENV. El pago de la matrícula o pensión aumenta en la escala de acuerdo al avance que se registra en el sistema. 
Cuadro 4. Porcentaje de personas desplazadas entre 5 y 17 años de edad que asisten a establecimientos educativos, por nivel educativo y según pagos que tienen que realizar, por condición de inscripción en el RUPD

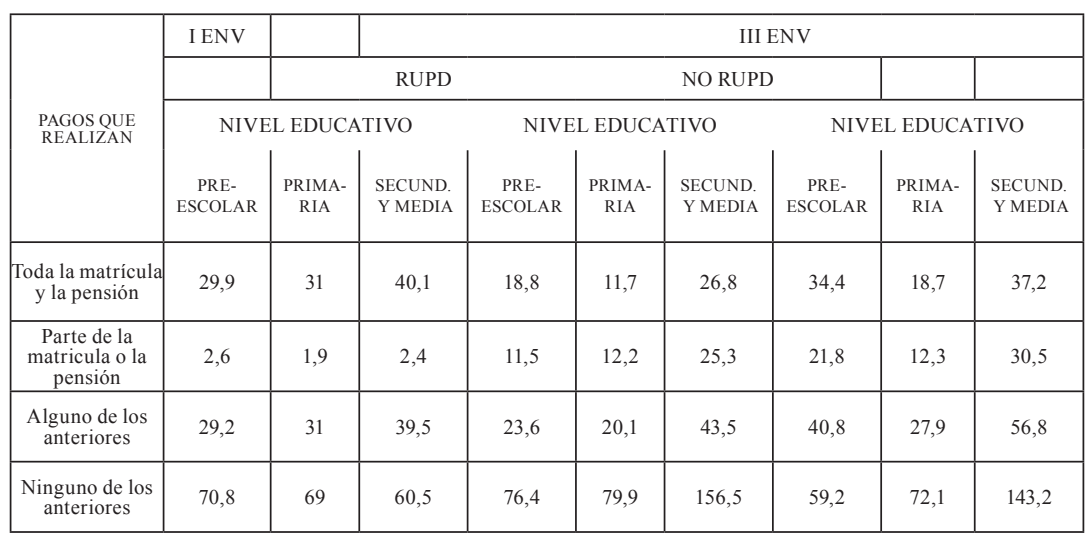

Fuente: En base a los resultados obtenidos por la I ENV-2007 y la III ENV-2010 Comisión de Seguimiento CID-UN

Cuarto, la Permanencia, que se expresa en que el $80,3 \%$ de la población entre 5-17 años asiste a preescolar, primaria, secundaria o media, mientras que el 18,9\% no asiste a ningún nivel educativo. La tasa de deserción intra-anual de niños entre 5- 11 años de edad es de 3,4\% y del 5\% para niños entre 12-15 años de edad; sin embargo, la tasa de deserción permanece alta (18,5\% para niños entre 15 y 16 años). Entre las causas de deserción se destacan: las de tipo económico (24\%), baja valoración dada a la educación $(21,8 \%)$ e incapacidad del sistema educativo (buscan cupo pero no encuentran $(20,9 \%)$.

Quinto, la Calidad, que se ve afectada por diferentes aspectos que rigen para la totalidad del sistema escolar y que, regularmente, acompañan y se intensifican en el ambiente escolar de sectores ampliamente populares, como la población desplazada. Uno de ellos, que afecta tanto el entorno como las instituciones mismas es el de la inseguridad; así, en la primera encuesta la inseguridad en el exterior del colegio contribuye, en un 33,8, \%, a la baja calidad de la educación. A ello se agrega el elevado número de estudiantes por salón $(29,6)$ y la falta de bibliotecas, laboratorios o salas de computo (27,7\%). El grado de realización del derecho a la educación, como se ha señalado, se ve afectado por múltiples razones, pero además, no se cumple la pretensión de que todos los niños y niñas en el hogar entre 5-17 años asistan regularmente a un nivel de educación formal. 


\section{El derecho a la educación en contextos regionales}

Uno de los dramas más comunes que acompañan el desarrollo individual de los niños y su proyección en la vida social en la modernidad es, sin lugar a dudas, el paulatino y temprano desarraigo al que se someten en los centros urbanos cuando los adultos -sus padres o familiares- deben engancharse rápidamente a los sistemas productivos, de tal forma que puedan responder a los avatares y exigencias propias del trabajo eficaz, que exige la sociedad opulenta y esnobista de nuestra época. Este "sacrificio", se realiza en virtud de un pretendido bienestar, que se supone debe extenderse -cuando ello se logra- a los menores de edad. En el caso de los niños, niñas y adolescentes desplazados, el problema es de mayor complejidad, pues el anclaje de sus padres y familiares a los sistemas productivos (proyectos de generación de ingresos), suele producirse en condiciones de mayor precariedad (escasos vínculos laborales y contractuales legales), en relación con el resto de la población. El resultado final es que un alto porcentaje de estos menores $(8,3 \%)$, no vive con ninguno de sus padres y el $34,7 \%$ adicional vive con uno sólo de los progenitores -en su altísima mayoría con la madre- lo cual no deja de exponerlos a distintos tipos de riesgo (abuso sexual, fichaje y seguimiento por parte de bandas emergentes y demás).

Sobre la base de estas premisas del orden nacional se puede, entonces, analizar los resultados de este trabajo a nivel territorial. Para el caso de la Población en Situación de Desplazamiento Forzado en el Área Metropolitana de Bucaramanga (AMB) es preciso señalar que ha sido foco de atención por parte de múltiples agencias, tanto nacionales como internacionales. No obstante, estos esfuerzos deben valorarse según el impacto sobre el bienestar y la calidad de vida en el corto, mediano y largo plazo, en el sentido señalado por Amartya Sen, Martha Nussbaum y otros.

En la encuesta realizada en el AMB con el apoyo del Nodo IEH (que se aplicó, según el modelo de la Encuesta Nacional de Verificación elaborado por la Comisión de Seguimiento a la Política Pública sobre Desplazamiento Forzado), en el ítem relacionado con el Derecho a la Educación se puede observar el alcance de la política de estabilización socioeconómica de esta población. La educación no es, ni más ni menos importante que los demás aspectos contemplados en la encuesta (salud, vivienda, seguridad alimentaria, reparación, entre otros), pero es fundamental para dar cuenta del efecto de las Políticas de Estabilización Socioeconómica promovidas, tanto por el Estado como por los Entes Territoriales que están al frente de esta ardua tarea.

En primer lugar, se pudo observar que, en el total de los hogares encuestados, los menores de 18 años que asisten a la escuela representan aproximadamente el 80\%, ,sin embargo, es alta la cifra de quienes se quedan por fuera del sistema escolar. 
Cuadro No.5 Asistencia Escolar en el AMB

\begin{tabular}{lccc}
\hline \multicolumn{1}{c}{ Asistencia } & Frecuencia & Porcentaje & Acumulado \\
\hline $\mathrm{Si}$ & 202 & 79,84 & 79,84 \\
$\mathrm{No}$ & 51 & 20,16 & 100 \\
Total & 253 & 100 & \\
\hline
\end{tabular}

Fuente: Encuesta adelantada por el Centro de Investigaciones Epidemiológicas de la UIS 2010

En segundo lugar, el 33\% de los menores de edad que asisten a los establecimientos educativos deben pagar matrícula y el $67 \%$ pensión mensual. Si se tienen en cuenta las precarias condiciones de acceso de los padres y jefes de hogar al mercado laboral, estos costos de la educación resultan una pesada carga para los hogares. Esto último se evidencia al revisar las cifras ya que sólo el 39\% de los jefes de hogar encuestados manifestaron estar ocupados y el $25 \%$ sólo han estado ocupados esporádicamente. El resto (36\%) no realizaban ninguna actividad, ni siquiera en el mercado informal. En el siguiente cuadro se registra un conjunto de aspectos que también dificultan, tanto el acceso como la permanencia en el sistema educativo (1. Libros y útiles, 2. Uniformes, 3. Transporte, 4. Implementos de Aseo, 5. Considera que no está en edad escolar o que ya terminó, 6. Buscó cupo pero no encontró, 7. Responsabilidades familiares, 8. Todas, 9. Ninguna). A manera de conclusión se puede afirmar que los costos de los útiles, uniformes y el transporte son los factores que mayor peso (70,84\%) tienen en este abanico de dificultades para acceder y permanecer en el sistema educativo entre las familias encuestadas en el AMB, lo cual confirma la percepción de que la política no genera caminos efectivos de integración cultural y social para quienes son víctimas del desplazamiento y el desarraigo.

Cuadro No.6 Acceso al sistema educativo en el AMB

\begin{tabular}{lccc}
\hline \multicolumn{1}{c}{ Dificultades } & Frecuencia & Porcentaje & Acumulado \\
\hline Libros y útiles & 109 & 49,32 & 49,32 \\
Uniformes & 27 & 12,22 & 61,54 \\
Transporte & 21 & 9,5 & 71,04 \\
Implementos de aseo & 1 & 0,45 & 71,49 \\
No está en edad escolar & 7 & 3,17 & 74,66 \\
Buscó cupo y no encontró & 8 & 3,62 & 78,28 \\
Responsabilidades familiares & 46 & 20,81 & 99,1 \\
Ninguna & 2 & 0,9 & 100 \\
Total & 221 & 100 & \\
\hline
\end{tabular}

Fuente: Encuesta adelantada por el Centro de Investigaciones Epidemiológicas de la UIS 
Como se dijo atrás, es notoria la precariedad de acceso al mercado de trabajo entre la población desplazada. Así, en calidad de obrero o empleado particular o del gobierno se ubica tan sólo el $12,55 \%$ de los jefes de hogar encuestados; en las posiciones de empleado doméstico, jornalero y peón se concentra el 23,5\%; como trabajador familiar sin remuneración y en calidad de rebusque se encuentra el $26 \%$, en tanto que el grueso de los mismos afirmó desempeñarse como trabajador independiente (38\%). Las cifras anteriores refuerzan, para el AMB, la conclusión de las ENV para el nivel nacional: son mínimas las oportunidades que encuentran los desplazados en los lugares de recepción, para conseguir puestos de trabajo estables que garanticen los recursos necesarios para ofrecer educación a los miembros del hogar de menor edad. Con ello, se reproducen y refuerzan las condiciones de vulnerabilidad de esta población.

Como se sabe, el ambiente escolar se constituye en una de las piezas claves del proceso educativo $\mathrm{y}$, por tanto, de la integración y estabilización socioeconómica de los segmentos poblacionales con menores oportunidades. En este sentido cabe señalar que, en el AMB, los menores de edad que asisten a preescolar, primaria y secundaria, perciben en los establecimientos educativos un conjunto de problemas entre los que merece la pena destacar: falta de profesores $(10 \%)$, instalaciones inadecuadas $(8,3 \%)$, inexistencia de bibliotecas $(6,14 \%)$, hacinamiento $(9,65 \%)$ e inseguridad $(9,21 \%)$. En ocasiones, se suma a estos factores la prevención frente a su condición de desplazamiento, que puede entrabar aún más el proceso de asimilación a los nuevos ambientes escolares.

Cuadro No.7 Percepción Ambiente Escolar

\begin{tabular}{lccc}
\hline \multicolumn{1}{c}{ Percepción del ambiente escolar } & Frecuencia & Porcentaje & Acumulado \\
\hline Falta de profesores & 23 & 10,09 & 10,09 \\
Instalaciones inadecuadas & 19 & 8,33 & 18,42 \\
Falta de biblioteca & 14 & 6,14 & 24,56 \\
Hacinamiento & 22 & 9,65 & 34,21 \\
Inseguridad & 21 & 9,21 & 43,42 \\
Cierres extemporáneos & 9 & 3,95 & 47,37 \\
Más de una de las anteriores & 116 & 50,88 & 98,25 \\
Ninguna & 4 & 1,75 & 100 \\
Total & 228 & 100 & \\
\hline
\end{tabular}

Fuente: Encuesta adelantada por el Centro de Investigaciones Epidemiológicas de la UIS, (2010)

En todo momento, se ha generado una tensión entre diversos grupos sociales e instituciones alrededor de aquello que se considera una política gubernamental incluyente, es decir, generadora de condiciones propicias para el goce efectivo de los derechos fundamentales en materia de salud vivienda y educación que, a la vez 
permita no sólo generar ingresos, sino construir un tejido social sólido a través de la participación. Esta tensión es mayor aún cuando se trata de fijar una política de Estado que vaya más allá de las acciones y prácticas discursivas generadas por el establecimiento alrededor de lo que se considera en nuestro país -desde hace algunos años- como la joya de la corona, esto es la llamada seguridad democrática.

Nos referimos de manera particular al tema de la reparación integral de las víctimas sometidas al despojo masivo de sus bienes materiales y simbólicos, en el marco de lo que se ha dado en denominar verdad, justicia y reparación, así como de las garantías de no repetición para el caso de la población desplazada. El proceso de verificación, adelantado por la comisión de seguimiento a la política pública sobre desplazamiento forzado ha permitido cuestionar la política pública, corregirla e incluso mejorarla de manera sustancial. A escala regional, este cuestionamiento también es posible para el tema del derecho a la educación ya que, sin desconocer los esfuerzos realizados en este ámbito a nivel territorial, existe todavía una multiplicidad de factores que inciden negativamente en la posibilidad de que los niños y adolescentes de los hogares en situación de desplazamiento, puedan acceder a una educación de calidad que reconozca sus problemáticas particulares y se convierta en elemento de cohesión y crecimiento personal y social.

\section{Conclusiones}

Las conclusiones se pueden derivar a partir de núcleos problemáticos centrales, con el propósito de llamar la atención de los hacedores de políticas en relación con aspectos que deben ser prioridad para la comunidad educativa en general y que resultan claves para entender el tipo de transformaciones que requiere el sistema educativo regional en su conjunto. Existe un consenso más o menos amplio entre los estudiosos del tema, en el sentido de considerar que, la educación como elemento central de la recuperación temprana, debe forjarse horizontes de planeación de largo plazo, so pena de enfrentarse a la coyuntura que sacude varios de los procesos educativos.

La crisis educativa y los procesos de la reforma educativa han generalizado la convicción de que el modelo tradicional de gestión educativa es más una parte del problema que un instrumento de solución del mismo, en particular cuando se trata de la población en situación de vulnerabilidad y riesgo. Esto se traduce en una continua frustración dentro y fuera del sistema educativo, y también en inconformismo por parte de los estudiantes, las familias y hogares de las personas en situación de desplazamiento quienes se sienten poco implicados en los procesos educativos.

Se hace necesario, entonces, continuar el proceso de re-conceptualizar la práctica educativa para alejarla, cada vez más, de la visión exclusivamente enciclopedista y normativa y comprometerla con el desarrollo de una concepción más amplia 
y significativa para todos los miembros de las comunidades afectadas por el desplazamiento forzado. Este reto es mucho más fuerte para los centros educativos locales, como un espacio adecuado y eficaz para aprender de todos los aspectos que involucra la vida cotidiana, incluido el aprendizaje comunitario y lograr, de esa forma, una educación para la vida.

Para el caso de la Población en Situación de Desplazamiento Forzado en el Área Metropolitana de Bucaramanga es preciso señalar que, estos conjuntos poblacionales han sido foco de atención por parte de múltiples agencias tanto nacionales como internacionales. No obstante, estos esfuerzos deben valorarse según el impacto sobre el bienestar y la calidad de vida en el corto, mediano y largo plazo, en el sentido señalado por Amartya Sen, Martha Nussbaum y otros.

Creemos importante destacar la visión de un grupo de líderes de Población en Situación de Desplazamiento Forzado del AMB que, al colaborar con esta iniciativa, ha pensado en el presente y futuro de sus hijos y familiares en condiciones de auténtica dignidad humana, sin etiquetas de ninguna naturaleza, sin exclusión social. El proyecto en curso se ha centrado en el análisis y discusión de la necesidad que tiene el Estado colombiano y la sociedad en su conjunto de abordar de manera radical y con carácter urgente el tema del despoblamiento que ha provocado la guerra en los territorios del país. En ese sentido, el análisis de los programas de estabilización socioeconómica se funda en un criterio más amplio que aquel que pretende tan sólo una mirada técnica de la evaluación de los programas del Gobierno. Se trata de una mirada a la complejidad del ordenamiento social producido por quienes hoy habitan este espacio y al carácter ético y político de las actividades que, como ciudadanos, se emprenden para restituir el daño causado a la sostenibilidad del territorio y a quienes lo habitan.

La educación, en sí misma, se constituye en un derecho que debe forjar las oportunidades necesarias para la inclusión digna a los subsistemas funcionales de la sociedad. Ello tiene un sentido preciso en las actuales circunstancias por las que atraviesa el país, al enfrentarse su población más vulnerable a un conjunto de emergencias complejas que llaman la atención de la comunidad humanitaria internacional. Niñas, niños y adolescentes y sus núcleos familiares, sometidos regularmente a un conjunto de desigualdades estructurales, afrontan ahora una multiplicidad de afectaciones, tanto por el conflicto interno y los impactos derivados del mismo, como por los fenómenos naturales que los golpean con mayor contundencia. Su problemática llama a una seria reflexión sobre el sentido de una educación incluyente, pertinente, con calidad, pero, sobre todo, capaz de constituirse en un factor de movilidad social y en herramienta propicia para la reconstitución de un tejido social fragmentado y excesivamente debilitado. 


\section{REFERENCIAS BIBLIOGRÁFICAS}

Bauman, Zygmunt (1999). La Globalización: consecuencias humanas. México, Fondo Cultura Económica.

Castoriadis, Cornelius (2004). Sujeto y verdad en el mundo histórico-social. Seminarios 1986-1987. Buenos Aires, Fondo de Cultura Económica de Argentina.

Mill, John Stuart (2001). Sobre la libertad. Madrid, Alianza Editorial.

Morin, Édgar y Motta, Raúl (2008). Educar en la era planetaria, Barcelona, Editorial Gedisa.

Naredo, J (1996). La economía en evolución: historias y perspectivas de las categorías básicas del pensamiento económico. Madrid, Siglo XXI Editores.

Nussbaum, Martha C (2007). Las fronteras de la justicia: consideraciones sobre la exclusión. Barcelona, Editorial Paidós.

Rawls, John (2005). La justicia como equidad: una reformulación.Barcelona, Editorial Paidós Estado y Sociedad.

Sala i Martín (1999). Apuntes del crecimiento económico. Barcelona. Anthony Bosch Editor.

Sarmiento, Palacio, Eduardo (2000). Como construir una nueva organización económica. Santa fe de Bogotá, Editorial Oveja Negra Ltda.

Sen, Amartya (2000). Desarrollo y Libertad. Barcelona, Editorial Planeta.

Solow, Robert (1992). La teoría del crecimiento. México, Fondo de Cultura Económica.

Vigotsky, Lev S (2005). La imaginación y el arte en la infancia. México D. F., Ediciones Coyoacán. 